\title{
NONVANISHING OF $L$-SERIES AND THE COMBINATORIAL SIEVE
}

\author{
JefFrey Hoffstein AND Wenzhi Luo \\ with an Appendix by \\ DAVID E. ROHRLICH
}

\section{Introduction}

Let $f(z)=\sum_{n=1}^{\infty} a_{n} e(n z)$ be a normalized holomorphic newform of weight $k \geq 2$, level $N$ with trivial nebentype as in [8], and let $L(s, f)$ be the associated Hecke $L$-function. For any fundamental discriminant $d,(d, N)=1$, we have the Kronecker symbol $\chi_{d}(\cdot)=(\underline{d})$, and the corresponding twisted $L$-function $L\left(s, f, \chi_{d}\right)$. It was proved by Hecke [5], by means of Mellin inversion, that the $L$-function $L\left(s, f, \chi_{d}\right)$ is an entire function of $s$ and satisfies the functional equation

$$
\begin{aligned}
& \left(\frac{|d| \sqrt{N}}{2 \pi}\right)^{s} \Gamma(s) L\left(s, f, \chi_{d}\right)= \\
& \quad w \chi_{d}(-N)\left(\frac{|d| \sqrt{N}}{2 \pi}\right)^{k-s} \Gamma(k-s) L\left(k-s, f, \chi_{d}\right),
\end{aligned}
$$

where $w= \pm 1$ is the root number. Thus $k / 2$ is at the center of the critical strip.

The central $L$-values $L\left(k / 2, f, \chi_{d}\right)$ are intimately related in a mysterious way to the form $f$ and in many cases they are known to have deep arithmetic meanings. For example one has the Birch-Swinnerton-Dyer conjecture, relating the arithmetic of a modular elliptic curve $E$ to the critical value of the associated Hasse-Weil L-function $L(E, s)=L(f, s)$ (where $f$ is a newform of weight 2 parametrizing $E$ ). Waldspurger [12], in his far-reaching works on the Shimura correspondence, first proved the nonvanishing of $L\left(k / 2, f, \chi_{d}\right)$ for infinitely many fundamental discriminants $d$.

In this note, we will establish the following refined nonvanishing result, by means of the combinatorial sieve and analytic methods.

Theorem. There exist infinitely many odd fundamental discriminants $d$ such that

$$
L\left(k / 2, f, \chi_{d}\right) \neq 0
$$

Received December 23, 1996.

Research supported in part by NSF Grants. 
and the number of prime factors of $d$ is no greater than 4. Moreover if $S$ is any finite set of rational primes then there exist infinitely many odd fundamental discriminants $d$ with the above properties and such that $\chi_{d}(p)=1$ for all $p \in S$.

Remark 1. For an interesting application of our theorem to exhibiting the unboundedness of the Tate-Shafarevich group in families of quadratic twists of any (modular) elliptic curve, see D.Rohrlich's appendix to this paper [11].

Remark 2. Although we omit the details, we note that by use of a weighted sieve this result can be further sharpened to the constraint that at most 3 distinct primes divide $d$. Because of the parity phenomenon of sieve methods it is not likely that our argument could be strengthened to extend to the case of $d$ prime. However one should still expect the result to be true in this case. See Ono's paper [9] for supporting examples.

\section{Proof of the theorem}

To present the main idea, we'll first prove the existence of an $r$ such that there exist infinitely many odd fundamental discriminants $d$ with $L\left(k / 2, f, \chi_{d}\right) \neq 0$, $\omega(d)=\sum_{p \mid d} 1 \leq r$ and such that $\chi_{d}(p)=1$ for all $p \in S$. This is done using only sieve techniques and a method of Iwaniec [7]. Then we'll indicate how an optimal $r$ can be obtained by making use of some properties of metaplectic forms described in [2].

Let $f$ be as in $\S 1$, assume $N \mid M$, where $M$ is divisible by any prime $p \in S$. and define

$\Delta^{ \pm}=\left\{d, \quad \pm d>0, \mu(d) \neq 0, d \equiv \nu^{2} \quad(\bmod 4 M)\right.$, for some $\nu$ coprime to $\left.4 M\right\}$.

Thus we'll have

$$
w \chi_{d}(-N)=1, \quad d \in \Delta^{w} .
$$

Let $F(\cdot)$ be a non-negative smooth function, compactly supported in $(0,1)$ with positive mean value. For $Y>3$, we set

$$
c_{n}= \begin{cases}L\left(k / 2, f, \chi_{n}\right) F(|n| / Y), & \text { if } n \in \Delta^{w} \\ 0, & \text { otherwise }\end{cases}
$$

Consider

$$
A=\sum_{n \in Z, \omega(n) \leq r} c_{n}
$$

To prove our theorem, it suffices to prove $A \gg Y / \log Y$ for sufficiently large $Y$. Since $c_{n} \geq 0$ (see [3] for example), we have

$$
A \geq \sum_{\left(n, P\left(Y^{1 /(r+1)}\right)\right)=1} c_{n}
$$

where $P(z)=\prod_{p<z} p$. 
A minor modification of Iwaniec's method [7] gives that for $d_{0} \mid P(z)$ and $\left(d_{0}, 4 M\right)=1$,

$$
\sum_{n \equiv 0} c_{n}=C_{f} \frac{\rho\left(d_{0}\right)}{d_{0}} Y+O\left(\sigma_{1}\left(d_{0}\right) d_{0}^{1 / 2} Y^{\frac{13}{14}+\epsilon}\right)
$$

where $C_{f} \neq 0$ is some constant depending on $f$ only, $\sigma_{1}\left(d_{0}\right)=\sum_{d \mid d_{0}} d$, and the constant implied in $O$-symbol depends on $\epsilon$ and $f$ only; $\rho\left(d_{0}\right)$ is a multiplicative function in $d_{0}$ :

$$
\rho\left(d_{0}\right)=\frac{\phi\left(d_{0}\right)}{d_{0}} \prod_{p \mid d_{0}}\left(1+\sum_{i \geq 1} a_{p^{2 i}}(1+1 / p)^{-1} p^{-k i}\right)^{-1},
$$

where $\phi\left(d_{0}\right)$ is the Euler function

$$
\phi\left(d_{0}\right)=d_{0} \prod_{p \mid d_{0}}(1-1 / p)
$$

Applying Deligne's bound [1]

$$
\left|a_{n}\right| \leq d(n) n^{(k-1) / 2}
$$

we conclude that

$$
\rho(p)=1+O(1 / p)
$$

Define

$$
r_{d}=\sum_{n \equiv 0(d)} c_{n}-C_{f} Y \frac{\rho(d)}{d} .
$$

Now, as in the Rosser-Iwaniec combinatorial sieve [4], for $z \leq \sqrt{D}$ we introduce the set

$\mathcal{D}=$

$\left\{d: d \mid P(z), d=1\right.$, or $p_{l} \cdots p_{1}, p_{l}<\cdots<p_{1}, p_{m}^{3} p_{m-1} \cdots p_{1}<D$, for $\left.m \leq l, 2 \mid m\right\}$,

and let $\theta(d)$ be the characteristic function of $\mathcal{D}$. Then we have ([4], pages 89-91)

$$
\sum_{d \mid n} \mu(d) \geq \sum_{d \mid n} \mu(d) \theta(d)
$$

and evidently

$$
\theta(d) \neq 0 \Rightarrow d \leq D
$$


From (4), we infer that

$$
\begin{aligned}
A & \geq \sum_{n} c_{n} \sum_{d \mid\left(n, P\left(Y^{1 /(r+1)}\right)\right)} \mu(d) \\
& \geq \sum_{n} c_{n} \sum_{d \mid\left(n, P\left(Y^{1 /(r+1)}\right)\right)} \mu(d) \theta(d) \\
& =\sum_{d \mid P\left(Y^{1 /(r+1)}\right)} \mu(d) \theta(d) \sum_{n \equiv 0(\bmod d)} c_{n} \\
& =\sum_{d \mid P_{4 M}\left(Y^{1 /(r+1)}\right)} \mu(d) \theta(d) \sum_{n \equiv 0(\bmod d)} c_{n},
\end{aligned}
$$

since $d \mid n, c_{n} \neq 0$ implies $(d, 4 M)=1$. Here $P_{4 M}(z)=\prod_{p<z,(p, 4 M)=1} p$.

From the definition of $r_{d}$, we obtain

$$
\begin{aligned}
\text { (6) } A & \geq \sum_{d \mid P_{4 M}\left(Y^{1 /(r+1)}\right)} \mu(d) \theta(d)\left(C_{f} Y \frac{\rho(d)}{d}+r_{d}\right) \\
\text { (7) } & =C_{f} Y \sum_{d \mid P_{4 M}\left(Y^{1 /(r+1)}\right)} \mu(d) \theta(d) \frac{\rho(d)}{d}+\sum_{d \mid P_{4 M}\left(Y^{1 /(r+1)}\right)} \mu(d) \theta(d) r_{d} .
\end{aligned}
$$

For $\mathcal{P}^{\prime}$ a set of primes, define

$$
P^{\prime}(z)=\prod_{p<z, p \in \mathcal{P}^{\prime}} p
$$

and

$$
P^{\prime}\left(z_{1}, z\right)=\prod_{z_{1} \leq p<z, p \in \mathcal{P}^{\prime}} p .
$$

We will make use of the following version of the Jurkat-Richert theorem for the linear sieve (see Lemma 3 in [6]):

Lemma. Let $\rho(d)$ be a multiplicative function satisfying

$$
0 \leq \frac{\rho(d)}{d}<1, \text { for } d \mid P^{\prime}(z)
$$

and for $2 \leq z_{1}<z$,

$$
\prod_{p \mid P^{\prime}\left(z_{1}, z\right)}(1-\rho(p) / p)^{-1} \leq\left(\log z / \log z_{1}\right)\left(1+B / \log z_{1}\right),
$$

with constant $B>1$. Then we have

$$
\begin{array}{r}
\sum_{d \mid P^{\prime}(z)} \mu(d) \theta(d) \frac{\rho(d)}{d} \geq \prod_{p \mid P^{\prime}(z)}(1-\rho(p) / p)\{f(\log D / \log z)+ \\
\left.O\left(e^{\sqrt{B}-\log D / \log z}(\log D)^{-1 / 3}\right)\right\}
\end{array}
$$


where $f(s)$ is a (strictly) monotonely increasing, continuous function for $s \geq 2$ with $f(2)=0$ and satisfying $f(s)=1+O\left(e^{-s}\right)$ as $s \rightarrow+\infty$. We have a similar upper bound if the symbol ' $\geq$ ' in (10) is replaced by ' $\leq$ ' and $f(s)$ by $F(s)$ as defined in $[6]$.

We now choose $\mathcal{P}^{\prime}$ to be the set of all primes not dividing $4 M$, for any $M$ which is a multiple of $N$, and easily verify conditions (8) and (9) by the well-known Mertens formula [10]

$$
\sum_{p<z} 1 / p=\log \log z+C+O(1 / z)
$$

Choosing $D=Y^{1 / 1000}$ and $r$ sufficiently large (hence $\log D / \log Y^{1 /(r+1)}$ is large), we deduce from (9) and (10) that

$$
\sum_{d \mid P_{4 M}\left(Y^{1 /(r+1)}\right)} \mu(d) \theta(d) \frac{\rho(d)}{d} \gg 1 / \log Y .
$$

On the other hand, we note that

$$
\begin{aligned}
\left|\sum_{d \mid P_{4 M}\left(Y^{1 /(r+1)}\right)} \mu(d) \theta(d) r_{d}\right| & \leq \sum_{d \mid P_{4 M}\left(Y^{1 /(r+1)}\right), d<Y^{1 / 1000}}\left|r_{d}\right| \\
& \ll Y^{13 / 14+\epsilon} \sum_{d<Y^{1 / 1000}} \sigma_{1}(d) d^{1 / 2} \ll Y^{14 / 15} .
\end{aligned}
$$

Hence

$$
\left|\sum_{d \mid P_{4 M}\left(Y^{1 /(r+1)}\right)} \mu(d) \theta(d) r_{d}\right| \ll Y^{14 / 15} .
$$

Thus we conclude, in view of the lemma, (6), (7), (11), and (12), that

$$
A=\sum_{\left(n, P\left(Y^{1 /(r+1)}\right)\right)=1} c_{n} \gg Y / \log Y
$$

This establishes the existence of $r$ in the theorem.

\section{Metaplectic techniques}

Applying the method in [2], we can improve (5) substantially on average and establish a result analogous to

$$
\sum_{d<D}\left|r_{d}\right| \ll Y^{1 / 2} D^{1+\epsilon}
$$

This will immediately imply that $D=Y^{1 /(2+4 \epsilon)}$ and $r=4$ are admissible. One can further sharpen this to $r=3$ by means of a weighted sieve. This requires both the lower and the upper bounds in the Lemma and we omit the details here. 
The main idea is to replace $c_{n}$ in (3) (without the weighting factor $F(|n| / Y)$ ) by a somewhat complicated but actually more natural expression:

$$
C_{n}=\sum_{m d^{2}=n} \prod_{p^{\gamma} \| d}\left(A_{p^{2 \gamma}}-\left(\frac{p}{m}\right) p^{-1 / 2} A_{p^{2 \gamma-1}}\right) L\left(k / 2, f, \chi_{m}\right),
$$

where $A_{n}=a_{n} n^{(1-k) / 2}$, and consider the Dirichlet series

$$
Z_{M}(s)=\sum_{(n, M)=1} \frac{C_{n}}{n^{s}}
$$

As observed in [2], $Z_{M}(s)$ is related to a Rankin-Selberg convolution of $f$ with certain half-integral weight Eisenstein series and from this the analytic properties of $Z_{M}(s)$ can be derived. Specifically, for any $M$ with $16 N \mid M$ let

$$
\begin{gathered}
E_{M}^{\infty}(z, s, k)=\frac{1}{2} \sum_{\gamma=\left(\begin{array}{ll}
a & b \\
c & d
\end{array}\right) \in \Gamma_{\infty} \backslash \Gamma_{0}(M)}(\operatorname{Im} \gamma z)^{s}\left(\frac{c}{d}\right) \epsilon_{d}(c z+d)^{-k / 2} \\
\end{gathered}
$$

as in [2], and

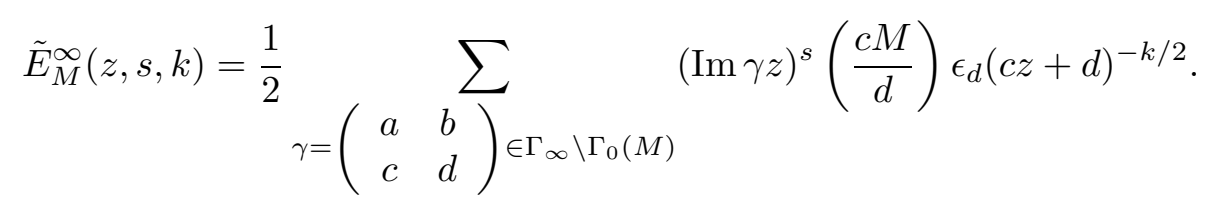

Expansions at the cusp 0 are given by

$$
E_{M}^{0}(z, s, k)=\left(M^{1 / 2} z\right)^{-k / 2} E_{M}^{\infty}(-1 / M z, s, k)
$$

and

$$
\tilde{E}_{M}^{0}(z, s, k)=\left(M^{1 / 2} z\right)^{-k / 2} E_{M}^{\infty}(-1 / M z, s, k) .
$$

If we then set

$$
F(s, M)=\int_{\Gamma_{0}(M) \backslash \mathcal{H}} \tilde{E}_{M}^{0}(z, s / 2,1) \overline{f(z)} E_{M}^{\infty}(z,(1-k+s) / 2,2 k-1) y^{k} \frac{d x d y}{y^{2}},
$$

unfold the Rankin-Selberg convolution and reverse the order of summation as in [2] we obtain

$$
F(s, M)=\frac{M^{-s / 2-1 / 4}}{\zeta_{M}(2 s)} Z_{M}(s) G(s),
$$

where $G(s)$ is a convolution of Whittaker functions that in this case reduces to a product of gamma factors and $\zeta_{M}(s)$ is the rational zeta function with Euler factors corresponding to primes dividing $M$ removed.

In order to obtain an improvement on (5) we consider the related Dirichlet series

$$
Z_{M}\left(s, M^{\prime}\right)=\left(M^{\prime}\right)^{s} \sum_{(n, M)=1, n \equiv 0\left(M^{\prime}\right)} \frac{C_{n}}{n^{s}}=\sum_{(n, M)=1} \frac{C_{n M^{\prime}}}{n^{s}}
$$


and note that for $\left(M, M^{\prime}\right)=1$

$$
\left(M^{\prime}\right)^{-s} Z_{M}\left(s, M^{\prime}\right)=\sum_{d \mid M^{\prime}} \mu(d) Z_{M d}(s)
$$

Thus $Z_{M}\left(s, M^{\prime}\right)$ can be constructed from the series $Z_{M d}(s)$ whose analytic properties are known. Added restrictions that the $n$ in the sum satisfy any given collection of congruence condition modulo primes dividing $M$ can be met by taking a linear combination of twists of $F(s, M)$ by characters with conductor dividing $M$. This is done by including characters in the definition of the Eisenstein series, as described in [2].

By standard Tauberian techniques, one can use these analytic properties and the residue at $s=1$ of $Z_{M}\left(s, M^{\prime}\right)$ to obtain estimates for the mean value of the $C_{n M^{\prime}}$. Briefly, for $s=1+\epsilon+i t, \epsilon>0$,

$$
Z_{M}(1+\epsilon+i t, d)=\sum_{(n, M)=1} \frac{C_{n d}}{n^{1+\epsilon+i t}}
$$

so

$$
\sum_{d<D}\left|Z_{M}(1+\epsilon+i t, d)\right| \leq \sum_{d<D} \sum_{(n, M)=1} \frac{d^{1+\epsilon} C_{n d}}{(n d)^{1}+\epsilon} \ll D^{1+\epsilon} \sum \frac{\sigma_{0}(m) C_{m}}{m^{1+\epsilon}} \ll D^{1+\epsilon}
$$

On the other hand,

$$
Z_{M}(-\epsilon-i t, d)=(M d)^{\epsilon+i t} \sum_{d^{\prime} \mid d} \mu\left(d^{\prime}\right) Z_{M d^{\prime}}(-\epsilon-i t)
$$

and after applying the scattering matrix for $F(s, M d)$ we obtain

$$
Z_{M d^{\prime}}(-\epsilon-i t) \ll\left(M d^{\prime}\right)^{1+\epsilon} \text {. }
$$

Thus

$$
Z_{M}(-\epsilon-i t, d) \ll d^{1+\epsilon},
$$

where the implied constant depends on $M, t$ (and is polynomial in both), and

$$
\sum_{d<D}\left|Z_{M}(-\epsilon-i t, d)\right| \ll D^{2+\epsilon}
$$

Note that many details have been suppressed in the computation of the scattering matrix and the upper bound as they are both routine and lengthy.

Applying convexity and moving to the center of the critical strip one obtains

$$
\sum_{d<D}\left|Z_{M}(1 / 2+i t, d)\right| \ll D^{3 / 2+\epsilon}
$$

If one sets

$$
R_{d}=\sum_{n \equiv 0(d)} C_{n} F(|n| / Y)-C_{f}^{\prime} Y \frac{\rho^{\prime}(d)}{d}
$$


having used the residue at $s=1$ of $Z_{M}(s, d)$ to evaluate the main term, one can then easily verify that the estimate of (14) above is an upper bound for $\sum_{d<D}\left|R_{d}\right|$, yielding the analog of (13).

\section{Acknowledgements}

The authors would like to thank David Rohrlich for bringing the above problem to their attention. Thanks are also due to Winnie Li for helpful discussions on this topic and to MSRI for excellent working conditions and financial support.

\section{References}

[1] P. Deligne, La conjécture de Weil I, Publ. Math. Inst. Hautes Etud. Sci. 43 (1974), 273-307.

[2] S. Friedberg and J. Hoffstein, Nonvanishing theorems for automorphic L-functions on GL(2), Ann. of Math. 142 (1995), 385-423.

[3] J. Guo, On the positivity of the central critical values of automorphic L-functions for GL(2), Duke Math. J. 83 (1996), 157-190.

[4] H. Halberstam and H.-E. Richert, Sieve methods, London Math. Soc. Monographs, 4, Academic Press, London-New York, 1974.

[5] E. Hecke, Über die Bestimmung Dirichletscher Reihen durch ihre Funktionalgleichung, Math. Ann. 112 (1936), 664-699.

[6] H. Iwaniec, A new form of the error term in the linear sieve, Acta Arith. XXXVII (1980), 307-320.

[7] - On the order of vanishing of modular L-functions at the critical point, Sém. Théor. Nombres Bordeaux (2) 2 (1990), 365-376.

[8] W. Li, Newforms and functional equations, Math. Ann. 212 (1975), 285-315.

[9] K. Ono, Twists of elliptic curves, Compositio Math., to appear, 1995.

[10] K. Prachar, Primzahlverteilung, Springer Verlag, Berlin-Göttingen-Heidelberg, 1957.

[11] D. Rohrlich, Appendix: Unboundedness of the Tate-Shafarevich group in families of quadratic twists, Math. Res. Lett. 4 (1997), 443-444.

[12] J.-L. Waldspurger, Sur les coefficients de Fourier des formes modulaires de poids demientier, J. Math. Pures Appl. 60 (1981), 375-484.

Department of Mathematics, Brown University, Providence, RI 02912 USA

E-mail address: jhoff@math.brown.edu

Department of Mathematics, Princeton University, Princeton, NJ 08544 USA

E-mail address: luo@math.princeton.edu 